\title{
OPEN MAPPINGS AND CLOSED SUBSETS OF THE DOMAIN IN GENERAL METRIC SPACES ${ }^{1,2,3}$
}

\section{JAMES EDGAR KEESLING}

Introduction. In the first part of this paper we prove the main result which gives sufficient conditions for an open mapping $f(X)=Y$ to have a closed set $K \subset X$ with $\operatorname{dim} K=0$ and $f(K)=Y$. This relates to a theorem of Civin and Roberts [1]. In the second section of the paper the main result is applied to an open mapping $f(X)=Y$ with $\operatorname{dim} X=m<\operatorname{dim} Y=n<\infty$ which states conditions under which there exists a $K$ closed in $X$ with $\operatorname{dim} K=0$ and $\operatorname{dim} f(K) \geqq n-m$. Several related results are also proved in this section. This is a continuation of the author's study of the relation of the dimension of closed subsets of the domain with the dimension of their image under various mappings [4].

Notation. Throughout, $f$ will denote a mapping from the metric space $X$ onto the metric space $Y$. By mapping is meant a continuous function. By $\operatorname{dim} X$ is meant the Lebesgue covering dimension of $X$. The pertinent background in dimension theory for general metric spaces will be found in Nagata [6]. Any additional assumptions on $f, X$, or $Y$ will be explicitly stated in each theorem.

I. $K$ closed in $X$ with $\operatorname{dim} K=0$ and $f(K)=Y$. All the results of this section relate to a paper of Civin and Roberts [1]. The main result is Theorem I.1.

I.1. Theorem. Let $f$ be open with (1) $\operatorname{dim} Y=n<\infty ;(2) f^{-1}(y)$ complete for all $y \in Y$; and (3) for every pair of disjoint closed sets $F_{1}$ and $F_{2}$ in $Y$ there is a closed set $B \subset Y$ separating $F_{1}$ and $F_{2}$ with the property that $f^{-1}(y)$ is perfect for all $y \in B$. Then there exists a $K \subset X$ with $K$ closed in $X$ and $\operatorname{dim} K=0$ and with $f(K)=Y$ and $f \mid K$ at most $n+1$ to one.

We will need a number of results before we can prove I.1. A remark is in order concerning condition (3) of the theorem. It could be replaced by either of the two following conditions which are equivalent:

Presented to the Society, January 25, 1968 under the title Open mappings and closed zero-dimensional sets; received by the editors August 24, 1967.

1 The author wishes to express his gratitude to Professor Edwin Duda for directing the research of this paper.

2 The author holds a NASA predoctoral fellowship at the University of Miami.

3 These results will form a part of the author's Doctoral Dissertation. 
$\left(3^{\prime}\right)$ the set $A=\left\{y \in Y: f^{-1}(y)\right.$ is not perfect $\}$ has dimension at most zero; or, $\left(3^{\prime \prime}\right)$ there is a $\sigma$-locally finite basis $\left\{V_{\alpha}\right\}$ for $Y$ such that for all $y \in \operatorname{Fr}\left(V_{\alpha}\right), f^{-1}(y)$ is perfect. We defer the proof of the equivalence of these conditions; however, the equivalence of $\left(3^{\prime}\right)$ and (3) will be used later in the paper. Let us now proceed to establish the auxiliary results to prove $I .1$.

1.2. Lemma. Let $f$ be open with $\operatorname{dim} Y=0$. Then, if $\epsilon>0$ and $U$ is open with $f(U)=Y$, there exists $\left\{U_{\alpha}: \alpha \in \Gamma\right\}$ a collection of open sets in $X$ and $\left\{V_{\alpha}: \alpha \in \Gamma\right\}$ an open partition of $Y$ such that (1) $\bar{U}_{\alpha} \subset U$; (2) $f\left(U_{\alpha}\right)$ $=V_{\alpha} ;$ and $(3) \delta\left(U_{\alpha}\right)<\epsilon$ for all $\alpha \in \Gamma$.

Proof. For each $x \in U$ let $U_{x}$ be open with $x \in U_{x}$ such that $\delta\left(U_{x}\right)<\epsilon$ and $\bar{U}_{x} \subset U$. Then $\left\{f\left(U_{x}\right): x \in U\right\}$ is an open cover of $Y$. Let $\left\{V_{\alpha}: \alpha \in \Gamma\right\}$ be an open partition refining $\left\{f\left(U_{x}\right)\right\}$. For each $\alpha$ let $U_{\alpha}=f^{-1}\left(V_{\alpha}\right) \cap U_{x_{\alpha}}$ where $V_{\alpha} \subset f\left(U_{x_{\alpha}}\right)$ for $x_{\alpha}$. Then the collection $\left\{U_{\alpha}: \alpha \in \Gamma\right\}$ has the required properties of the lemma.

I.3. THEOREM. If $f$ is open with complete point inverses and $\operatorname{dim} Y=0$, then, if $U$ is open in $X$ with $f(U)=Y$, there is a $K \subset U, K$ closed, with $f \mid K$ a homeomorphism onto $Y$.

Proof. Let $\epsilon_{i} \rightarrow 0$ be a sequence of positive numbers. By applying Lemma I.2 inductively we obtain a sequence of open collections $\left\{U_{\alpha, i}: \alpha \in \Gamma_{i}\right\}$ and a corresponding sequence of open partitions of $Y$, $\left\{V_{\alpha, i}: \alpha \in \Gamma_{i}\right\}$, such that (1) $\bar{U}_{\alpha, i} \subset U$ for all $\alpha$ and $i$; (2) $f\left(U_{\alpha, i}\right)=V_{\alpha, i}$; (3) $\left\{V_{\alpha, i+1}\right\}<\left\{V_{\alpha, i}\right\}$; (4) $\delta\left(U_{\alpha, i}\right)<\epsilon_{i}$; and (5) $\left\{\bar{U}_{\alpha, i+1}\right\}<\left\{U_{\alpha, i}\right\}$. We then define $K=\bigcap_{i=1}^{\infty} \cup\left\{\bar{U}_{\alpha, i}: \alpha \in \Gamma_{i}\right\}$. For each $i$ the collection $\left\{U_{\alpha, i}\right\}$ is locally finite since $\left\{f^{-1}\left(V_{\alpha, i}\right)\right\}$ is. Therefore $U\left\{\bar{U}_{\alpha, i}: \alpha \in \Gamma_{i}\right\}$ is closed and $K$ is closed. Now let $y \in Y$. Then for each $i$ there is a unique $U_{\alpha, i}^{v}$ such that $f^{-1}(y) \cap U_{\alpha, i}^{\nu} \neq \Phi$. Since $\bar{U}_{\alpha, i+1}^{v} \subset U_{\alpha, i}^{v}$ and $\delta\left(U_{\alpha, i}\right)<\epsilon_{i}$ for all $i$, by the completeness of $f^{-1}(y)$ we must have $\bigcap_{i=1}^{\infty}\left[f^{-1}(y) \cap \bar{U}_{\alpha, i}^{v}\right]=f^{-1}(y) \cap K$ consist of a single point. Thus $f \mid K$ is one to one onto $Y$. It remains only to show that $f \mid K$ is open. If we let $B_{\alpha, i}=U_{\alpha, i} \cap K$ then $\left\{B_{\alpha, i}\right\}$ is a basis for $K$. One can easily see that $f\left(B_{\alpha, i}\right)=V_{\alpha, i}$ and thus $f \mid K$ is open.

I.4. TheOREM. Let $f$ be open with complete point inverses with $\operatorname{dim} Y=n<\infty$. Then, if $U$ is any open set in $X$ with $f(U)=Y$, there is a $K$ closed in $X, K \subset U$ with $f(K)=Y$ such that $\operatorname{dim} K \leqq n$ and $f \mid K$ is at most $n+1$ to one.

Proof. By Theorem III.8 in Nagata $[6$, p. 78] there is a space $Z$ and a closed mapping $g(Z)=Y$ such that $\operatorname{dim} Z=0$ and $g^{-1}(y)$ has at most $n+1$ points for all $y \in Y$. Let $A=\{(x, z): x \in X$ and $z \in Z$ 
and $f(x)=g(z)\}$. Recall that $A$ is closed in $X \times Z$. Let $\Pi_{1}$ and $\Pi_{2}$ be the restrictions to $A$ of the projections onto $X$ and $Z$, respectively. Then $\Pi_{1}(A)=X$ is closed since $g$ is closed with compact point inverses and $\Pi_{2}(A)=Z$ is open since $f$ is open. Let $U \subset X$ be as in the theorem. Then $V=\Pi_{1}^{-1}(U)$ is open in $A$ and $\Pi_{2}(V)=Z$. Applying I.3 we get a closed set $B \subset V$ such that $\Pi_{2} \mid B$ is a homeomorphism of $B$ onto $Z$. Thus $\operatorname{dim} B=0$ and $\Pi_{2} \mid B$ is one to one. Since $g$ is at most $n+1$ to one we must have $\Pi_{1} \mid B$ at most $n+1$ to one. Let $K=\Pi_{1}(B)$. Since $\Pi_{1} \mid B$ is again closed we must have $\operatorname{dim} K \leqq n$. Also clearly $f(K)=Y, K \subset U$, and $f \mid K$ is at most $n+1$ to one.

I.5. Theorem. In I.4 if $Y$ is compact, then $K$ can be required to be compact.

Proof. The space $Z$ will then be compact. Thus $B \sim Z$ will be also. But then $\Pi_{1}(B)=K$ must also be compact.

The next result is the main tool in proving Theorem I.1.

I.6. Lemma. Suppose $f$ is open and that conditions (1), (2), and (3) of $\mathrm{I} .1$ are satisfied. Let $\epsilon>0$ and let $\left\{U_{\gamma}: \gamma \in \Gamma\right\}$ be a collection of open sets in $X$ having the following properties:

(i) $\left\{\bar{U}_{\gamma}\right\}$ is a disjoint collection; (ii) $f\left(\cup_{\gamma \in \Gamma} U_{\gamma}\right)=Y$; (iii) for all $y \in Y, f^{-1}(y) \cap \bar{U}_{\gamma}=\Phi$ except for at most $n+1$ distinct $\gamma^{\prime} s$; and (iv) $\left\{\bar{U}_{\gamma}\right\}$ is locally finite. Then there is $\left\{V_{\alpha}: \alpha \in A\right\}$, a collection of open sets in $X$, such that $\left\{V_{\alpha}\right\}$ has properties (i), . ., (iv) and in addition satisfies (v) $\delta\left(V_{\alpha}\right)<\epsilon$ for all $\alpha$ and (vi) for all $\alpha, \vec{V}_{\alpha} \subset U_{\gamma}$ for some $\gamma$.

PRoof. For each $x \in U_{\gamma \in \Gamma} U_{\gamma}$ let $U_{x}$ be open such that $\delta\left(U_{x}\right)<\epsilon$ and $x \in U_{x} \subset \bar{U}_{x} \subset U_{\gamma}$ for the unique $\gamma$ with $x \in U_{\gamma}$. Then $\left\{f\left(U_{x}\right)\right.$ : $\left.x \in \mathrm{U}_{\gamma \in \Gamma} U_{\gamma}\right\}$ is an open cover of $Y$. Let $\left\{W_{\alpha}: \alpha \in A\right\}$ be a locally finite refinement of order at most $n+1$ by the corollary on page 23 of Nagata [6]. Let $x_{\alpha}$ be such that $W_{\alpha} \subset f\left(U_{x_{\alpha}}\right)$ and define $V_{\alpha}^{\prime}$ $=f^{-1}\left(W_{\alpha}\right) \cap U_{x_{\alpha}}$. Note at this point that the collection $\left\{V_{\alpha}^{\prime}\right\}$ satisfies (ii), $\cdots$, (vi). The rest of the proof is concerned with finding $V_{\alpha} \subset V_{\alpha}^{\prime}$ such that $\left\{V_{\alpha}\right\}$ has all of the desired properties. Let $\left\{F_{\alpha}: \alpha \in A\right\}$ be a closed cover of $Y$ such that $F_{\alpha} \subset W_{\alpha}$ for all $\alpha \in A$. Let $U_{\alpha}$ be such that $F_{\alpha} \subset U_{\alpha} \subset \bar{U}_{\alpha} \subset W_{\alpha}$ for all $\alpha$ and such that for all $y \in \operatorname{Fr}\left(U_{\alpha}\right), f^{-1}(y)$ is perfect. This is possible by condition (3) of the lemma. Let $A$ be well ordered. Now $f \mid f^{-1}\left(\bar{U}_{\alpha_{1}}\right)$ is an open mapping onto $\bar{U}_{\alpha_{1}}$. Let $B_{\alpha_{1}}=f^{-1}\left(\bar{U}_{\alpha_{1}}\right) \cap V_{\alpha_{1}}^{\prime}$. Then $B_{\alpha_{1}}$ is open in $f^{-1}\left(\bar{U}_{\alpha_{1}}\right)$ and $f\left(B_{\alpha_{1}}\right)=\bar{U}_{\alpha_{1}}$. By Theorem I.4 we can get a $K_{\alpha_{1}} \subset B_{\alpha_{1}}$ closed in $f^{-1}\left(\bar{U}_{\alpha_{1}}\right)$, hence in $X$, such that $f\left(K_{\alpha_{1}}\right)=\bar{U}_{\alpha_{1}}$ and $f \mid K_{\alpha_{1}}$ is at most $n+1$ to one. Now suppose that we have constructed a collection of closed sets $\left\{K_{\alpha}: \alpha<\beta\right\}$ for $\beta \in A$ with the following properties: (1) for $\tau<\beta$ in $A$, 
$f\left(\mathrm{U}_{\alpha \leqq \tau} K_{\alpha}\right)=\mathrm{U}_{\alpha \leqq \tau} \bar{U}_{\alpha} ;$ (2) $K_{\alpha} \subset f^{-1}\left(\bar{U}_{\alpha}\right) \cap V_{\alpha}^{\prime} ;$ (3) $\left\{K_{\alpha}: \alpha<\beta\right\}$ is a disjoint collection; and (4) $f \mid K_{\alpha}$ is at most $n+1$ to one. We define $V_{\beta}^{\prime \prime}=V_{\beta}^{\prime}-U_{\alpha<\beta} K_{\alpha}$. Since $K_{\alpha} \subset V_{\alpha}^{\prime}, \quad\left\{K_{\alpha}: \alpha<\beta\right\}$ is locally finite. Therefore $U_{\alpha<\beta} K_{\alpha}$ is closed and $V_{\beta}^{\prime \prime}$ is open in $X$. Now let $y \in\left(\bar{U}_{\beta}-U_{\alpha<\beta} \bar{U}_{\alpha}\right)^{-}=G_{\beta}$. Now if $y \notin \bigcup_{\alpha<\beta} \bar{U}_{\alpha}$ then $f^{-1}(y) \cap K_{\alpha}=\Phi$ for all $\alpha<\beta$. Thus $f^{-1}(y) \cap V_{\beta}^{\prime \prime}=f^{-1}(y) \cap V_{\beta}^{\prime} \neq \Phi$. If $y \in G_{\beta}$ and $y \in U_{\alpha<\beta} \bar{U}_{\alpha}$, then $y \notin U_{\alpha}$ for any $\alpha<\beta$. Therefore $y \in \operatorname{Fr}\left(U_{\alpha}\right)$ for some $\alpha$ and thus $f^{-1}(y)$ is perfect. Now $f^{-1}(y) \cap \bigcup_{\alpha<\beta} K_{\alpha}$ is a finite set (with at most $n(n+1)$ elements). Of course $f^{-1}(y) \cap V_{\beta}^{\prime}$ is not finite. Thus $f^{-1}(y) \cap V_{\beta}^{\prime \prime} \neq \Phi$ in this case also. Let $K_{\beta} \subset V_{\beta}^{\prime \prime}$ be closed such that $f\left(K_{\beta}\right)=G_{\beta}$ and $f \mid K_{\beta}$ is at most $n+1$ to one. Then the collection $\left\{K_{\alpha}: \alpha<\beta+1\right\}$ has properties (1), . , (4) above. By transfinite induction we get a collection of closed sets $\left\{K_{\alpha}: \alpha \in A\right\}$ having properties (1), . , (4). Now define open sets $V_{\alpha}$ such that $K_{\alpha} \subset V_{\alpha} \subset V_{\alpha}^{\prime}$ such that the collection $\left\{\bar{V}_{\alpha}: \alpha \in A\right\}$ is a disjoint collection. Then $\left\{V_{\alpha}\right\}$ satisfies (i), $\cdots$, (vi) of the lemma.

We are now ready for the proof of I.1.

Proof OF I.1. Let $\epsilon_{i} \rightarrow 0$ be a sequence of positive numbers. Then let $\left\{V_{\alpha, i}: \alpha \in \Gamma_{i}\right\}$ be collections of open sets in $X$ having the following properties: (1) for each $i,\left\{\bar{V}_{\alpha, i}: \alpha \in \Gamma_{i}\right\}$ is a disjoint collection; (2) for each $i, f\left(\bigcup_{\alpha \in \Gamma_{i}} V_{\alpha, i}\right)=Y$; (3) for each $i, f^{-1}(y) \cap \bar{V}_{\alpha, i}=\Phi$ except for at most $n+1 \alpha$ 's in $\Gamma_{i}$; (4) $\left\{V_{\alpha, i}: \alpha \in \Gamma_{i}\right\}$ is locally finite; (5) $\delta\left(V_{\alpha, i}\right)<\epsilon_{i}$ for all $\alpha \in \Gamma_{i}$; and (6) $\left\{\bar{V}_{\alpha, i+1}: \alpha \in \Gamma_{i+1}\right\}<\left\{V_{\alpha, i}: \alpha \in \Gamma_{i}\right\}$. That such a sequence of collections exist is assured by the previous lemma and by induction. Let $K_{i}=\bigcup\left\{\bar{V}_{\alpha, i}: \alpha \in \Gamma_{i}\right\}$ and then let $K=\bigcap_{i=1}^{\infty} K_{i}$. Then $K$ is closed. Now let $B_{\alpha, i}=K \cap V_{\alpha, i}$. Then $\left\{B_{\alpha, i}\right\}$ is $\sigma$-locally finite in $K$. But $B_{\alpha, i}=K \cap \bar{V}_{\alpha, i}$ since $\operatorname{Fr}\left(V_{\alpha, i}\right) \cap K_{i+1}=\Phi$. Thus $B_{\alpha, i}$ is open and closed in $K$. Therefore $\operatorname{dim} K=0$. Now by (1), (6), and (3) above, for any $y \in Y$ we can easily obtain a sequence $\bar{V}_{\alpha, i}^{v}$ such that $\bar{V}_{\alpha, i+1}^{v} \subset \bar{V}_{\alpha, i}^{v}$ for all $i$, and $f^{-1}(y) \cap \bar{V}_{\alpha, i}^{v} \neq \Phi$. Since $\delta\left(\bar{V}_{\alpha, i}^{v}\right) \rightarrow 0$ we must have $f^{-1}(y) \cap\left[\bigcap_{i=1}^{\infty} \bar{V}_{\alpha, i}^{v}\right]=x$, a single point in $X$. But then $x \in K$ and $f(K)=Y$. Now suppose that there is a point $y \in Y$ with $\left\{x_{1}, \cdots, x_{n+2}\right\} \subset f^{-1}(y) \cap K$. Then let $\epsilon_{i}$ be small enough that $\left\{S\left(x_{j}, \epsilon_{i}\right): j=1, \cdots, n+2\right\}$ is a disjoint collection in $X$. But then $\bar{V}_{\alpha_{j}, i} \subset S\left(x_{j}, \epsilon_{i}\right)$ for the unique $\bar{V}_{\alpha_{j}, i}$ containing $x_{j}$. But then there are $n+2$ distinct $\alpha$ 's in $\Gamma_{i}$ with $f^{-1}(y) \cap \bar{V}_{\alpha, i} \neq \Phi$, a contradiction. Thus $f \mid K$ is at most $n+1$ to one and $K$ has now been shown to have all of the desired properties.

II. $K$ closed in $X$ with $\operatorname{dim} K=0$ and $\operatorname{dim} f(K)>0$. In this section we apply I.1 to open mappings under certain conditions to show the existence of closed 0-dimensional subsets of $X$ whose image must have 
dimension at least a certain positive value. This is a continuation of the author's work in the first part of [4].

II.1. Definition. $f$ is said to be $\sigma$-closed provided $X=\bigcup_{i=1}^{\infty} A_{i}$ where $A_{i}$ is closed in $X, f\left(A_{i}\right)$ is closed in $Y$, and $f \mid A_{i}: A_{i} \rightarrow f\left(A_{i}\right)$ is a closed mapping. $f$ is said to be dimension preserving on closed subsets provided that if $K$ is closed in $X$, then $\operatorname{dim} K=\operatorname{dim} f(K)$.

The author has shown in [4] that if $f$ is open and $\sigma$-closed with discrete point inverses then $f$ is dimension preserving on closed subsets. This result is sharpened in the next theorem and will be applied several times in the sequel.

II.2. THEOREM. If $f$ is open and $\sigma$-closed and $A=\{x \in X$ : $\left.x \in\left(f^{-1} f(x)-x\right)^{-}\right\}$with $X_{0}=X-A$, then $X_{0}$ is an $F_{\sigma}$ and $f \mid X_{0}: X_{0}$ $\rightarrow f\left(X_{0}\right)$ is dimension preserving on closed subsets.

Proof. Let $F_{n}=\left\{x \in X: d\left(x, f^{-1} f(x)-x\right) \geqq 1 / n\right\}$. By the openness of $f, F_{n}$ must be closed in $X$. But $X_{0}=\bigcup_{n=1}^{\infty} F_{n}$ and thus $X_{0}$ is an $F_{\sigma}$. Let $g \equiv f \mid X_{0}: X_{0} \rightarrow f\left(X_{0}\right)$. Then $g$ is also $\sigma$-closed since $X_{0}$ is an $F_{\sigma}$. Now $g$ is 0 -dimensional and so cannot lower the dimension of any closed subset of $X_{0}$. If $g$ is not dimension preserving on closed subsets, then by Corollary I.7 of [4], there is a closed set $K \subset X_{0}$ with $\operatorname{dim} K=0$ and $\operatorname{dim} g(K)>0$. Let $X=\cup_{i=1}^{\infty} A_{i}$ be as in the definition of $\sigma$-closed. We may assume $A_{i} \subset A_{i+1}$ for all $i$. By the definition of $F_{n}$ we have $F_{n} \subset F_{n+1}$ for all $n$. Let $B_{n}=F_{n} \cap A_{n}$. Then $B_{n}$ is closed in $X$ and $g \mid B_{n}: B_{n} \rightarrow g\left(B_{n}\right)$ is closed. Clearly $X_{0}=\cup_{n=1}^{\infty} B_{n}$. Now if $g(K)$ has positive dimension, then it is clear by the sum theorem for closed sets that there is an $n$ such that $\operatorname{dim} g\left(K \cap B_{n}\right)>0$. Let us suppose therefore that $K \subset B_{n}$. Let $\left\{Q_{\alpha}: \alpha \in \Gamma\right\}$ be a locally finite open cover of $X$ with $\delta\left(Q_{\alpha}\right)<1 / n$ for all $\alpha \in \Gamma$. Let $\left\{F_{\alpha}: \alpha \in \Gamma\right\}$ be a closed cover of $X$ with $F_{\alpha} \subset Q_{\alpha}$ for all $\alpha$. Let $K_{\alpha}=\left\{x \in F_{\alpha} \cap K: f^{-1} f(x) \cap Q_{\alpha}=x\right\}$. Then $f \mid K_{\alpha}$ is a homeomorphism onto $f\left(K_{\alpha}\right)$. Thus $\operatorname{dim} K_{\alpha}=\operatorname{dim} f\left(K_{\alpha}\right)$ for all $\alpha$. Now $\left\{K_{\alpha}: \alpha \in \Gamma\right\}$ is locally finite. Thus if $\Lambda \subset \Gamma$ is any subset then $L=\bigcup\left\{K_{\alpha}: \alpha \in \Lambda\right\}$ is closed. But $L \subset K \subset B_{n} \subset A_{n}$. Thus $f(L)$ $=g(L)$ is closed. But $f\left(\bigcup_{\alpha \in \Delta} K_{\alpha}\right)=\bigcup_{\alpha \in \Delta} f\left(K_{\alpha}\right)$. Thus we can apply a sum theorem [8, Corollary, p. 18] to $\left\{f\left(K_{\alpha}\right): \alpha \in \Gamma\right\}$ to get that $\operatorname{dim} \bigcup_{\alpha \in \Gamma} f\left(K_{\alpha}\right)=\sup _{\alpha \in \Gamma} \operatorname{dim} f\left(K_{\alpha}\right)=\sup _{\alpha \in \Gamma} \operatorname{dim} K_{\alpha}=\operatorname{dim} \bigcup_{\alpha \in \Gamma} K_{\alpha}$. But if $x \in K$, then since $K \subset F_{n}, x \in K_{\alpha}$ for some $\alpha$. Thus $\mathrm{U}_{\alpha \in \Gamma} K_{\alpha}=K$. That is, $\operatorname{dim} K=\operatorname{dim} f(K)=0$, a contradiction. Therefore $g$ must be dimension preserving on closed subsets.

The reader will observe that the only use made of the function $f$ being $\sigma$-closed was to get $g$ to be $\sigma$-closed. In case $X$ is separable this occurs as a result of the openness of $f$. Thus we can state the following improvement for $X$ separable. 
II.3. TheOREM. If $f$ is open and $X$ is separable with $A$ and $X_{0}$ as in II.2, then $f \mid X_{0}$ is $\sigma$-closed and dimension preserving on closed subsets onto $f\left(X_{0}\right)$ with $X_{0}$ and $f\left(X_{0}\right) F_{\sigma}$ 's in $X$ and $Y$, respectively.

Proof. Let $\left\{V_{i}\right\}_{i=1}^{\infty}$ be a basis for $X$ and let $B_{i}=\{x \in X$ : $\left.f^{-1} f(x) \cap V_{i}=x\right\}$. Then $B_{i}$ is an $F_{\sigma}$ and $f\left(B_{i}\right)$ is an $F_{\sigma}$ by the openness of $f$. Now $X_{0}=\bigcup_{i=1}^{\infty} B_{i}$ and $f\left(X_{0}\right)=\bigcup_{i=1}^{\infty} f\left(B_{i}\right)$. Thus these sets are $F_{\sigma}$ 's in their respective spaces. Now $f \mid B_{i}$ is a homeomorphism, hence closed onto $f\left(B_{i}\right)$. Therefore by Lemma I.3 of [6] $f \mid X_{0}$ is $\sigma$-closed. If $K$ is closed in $X_{0}$, then $\operatorname{dim} K \cap B_{i}=\operatorname{dim} f\left(K \cap B_{i}\right)$ and these sets are $F_{\sigma}$ 's. Thus we must have $\operatorname{dim} K=\operatorname{dim} f(K)$ and thus $f \mid X_{0}$ is dimension preserving on closed subsets.

The following lemma is necessary before we can proceed to the main results of this section.

II.4. Lemma. Let $A \subset X$ with $\operatorname{dim} A=m<\operatorname{dim} X=n$. Then there is a closed set $K \subset X$ with $\operatorname{dim} K \geqq n-1$ and $\operatorname{dim} K \cap A \leqq m-1$.

Proof. Let $A=\bigcup_{i=0}^{m} A_{i}$ with $\operatorname{dim} A_{i}=0$ for all $i$. There is a $\sigma$-locally finite basis for $X$, call it $\left\{V_{\alpha}: \alpha \in \Gamma\right\}$ such that $\operatorname{Fr}\left(V_{\alpha}\right) \cap A_{0}=\Phi$ for all $\alpha \in \Gamma$. Since $\operatorname{dim} X=n$, there is an $\alpha_{0}$ such that $\operatorname{dim} \operatorname{Fr}\left(V_{\alpha_{0}}\right) \geqq n-1$. But since $\operatorname{Fr}\left(V_{\alpha_{0}}\right) \cap A \subset \bigcup_{i=1}^{m} A_{i}$ we must have $\operatorname{Fr}\left(V_{\alpha_{0}}\right) \cap A$ having dimension at most $m-1$. Letting $K=\operatorname{Fr}\left(V_{\alpha_{0}}\right)$ we get our required set.

II.5. Lemma. Let $A \subset X$ with $\operatorname{dim} A=m<\operatorname{dim} X=n$. Then there is a closed set $K \subset X$ with $\operatorname{dim} K \geqq n-m$ and $\operatorname{dim} K \cap A \leqq 0$.

Proof. We apply Lemma II.4 inductively to get the required set.

We are now ready for the main result of this section.

II.6. THEOREM. If $f$ is open and $\sigma$-closed with complete point inverses with $\operatorname{dim} X=m<\operatorname{dim} Y=n<\infty$, then there is a closed set $K \subset X$ with $\operatorname{dim} K=0$ and with $\operatorname{dim} f(K) \geqq n-m$.

Proof. Let $A=\left\{x \in X: x \in\left(f^{-1} f(x)-x\right)^{-}\right\}$and $X_{0}=X-A$. Then $X_{0}$ is an $F_{\sigma}$ in $X$ and $f \mid X_{0}$ is dimension preserving on closed subsets. Thus $\operatorname{dim} f\left(X_{0}\right) \leqq m$. Let $F$ be closed in $Y$ with $\operatorname{dim} F \geqq n-m$ and $\operatorname{dim} F \cap f\left(X_{0}\right) \leqq 0$ by Lemma II.5. Now $g \equiv f \mid f^{-1}(F)$ is an open mapping onto $F$ with complete point inverses. Furthermore, $g$ satisfies the hypotheses of $I .1$ and thus there is a $K$ closed in $X$ with $\operatorname{dim} K=0$ and with $g(K)=f(K)=F$.

II.7. THEOREM. If $f$ is open with complete point inverses where $X$ is separable and $\operatorname{dim} X=m<\operatorname{dim} Y=n<\infty$, then there is a $K \subset X$, $K$ closed with $\operatorname{dim} K=0$ and with $\operatorname{dim} f(K) \geqq n-m$. 
Proof. Same as for II.6.

II.8. TheOREM. If $f$ is open and closed with $\operatorname{dim} X=m<\operatorname{dim} Y$ $=n<\infty$, then there is a $K \subset X$ with $\operatorname{dim} K=0, K$ closed, and $\operatorname{dim} f(K)$ $\geqq n-m$.

Proof. Let $B=\bigcup_{y \in Y} \operatorname{Fr}\left(f^{-1}(y)\right)$. Then $\operatorname{dim} B \leqq m$. Also we must have $\operatorname{dim} f(B)=n$. Now $B$ is an inverse set and thus $f \mid B: B \rightarrow f(B)$ is open and closed. But $f \mid B$ has compact point inverses. We apply II. 6 to $f \mid B$ to get a $K \subset B, K$ closed with $\operatorname{dim} K=0$ and with $\operatorname{dim} f(K)$ $\geqq n-m$. But $B$ is closed in $X$, thus $K$ is closed in $X$.

II.9. REMARK. One can consider II.6 to be a converse to a theorem due to Hurewicz which states that for closed $f$ with $\operatorname{dim} X=m$ $>\operatorname{dim} Y=n$, there is a $y \in Y$ with $\operatorname{dim} f^{-1}(y) \geqq m-n$.

With some extra effort we can find a surprising improvement of II.7 in case $X$ is a manifold.

II.10. ThEOREM. Let $f$ be open and $X$ a (separable) m-dimensional Euclidean manifold with $m<\operatorname{dim} Y=n<\infty$. Then there is a $K \subset X$, $K$ closed (compact) with $\operatorname{dim} K=0$ and $\operatorname{dim} f(K) \geqq n-m+1$.

Proof. Let $X$ have a complete metric. Then $f$ will have complete point inverses in this metric. Let $Y^{\prime}=Y-U\{U: U$ is open in $Y$ with $\operatorname{dim} U<n\}$. Then $Y^{\prime}$ is closed in $Y$ with $\operatorname{dim} Y^{\prime}=n$. Now consider $f \mid f^{-1}\left(Y^{\prime}\right)$ mapping on to $Y^{\prime}$. This mapping is open with complete point inverses. Let $B=\left\{x \in f^{-1}\left(Y^{\prime}\right): x \notin\left(f^{-1} f(x)-x\right)^{-}\right\}$. Then $B$ is an $F_{\sigma}$ in $f^{-1}\left(Y^{\prime}\right)$ and $f \mid B$ is dimension preserving on closed subsets. Now if $\operatorname{dim} B=m$, then there is an open $m$-cell $K^{m} \subset B$. But then $\operatorname{dim} K^{m}=\operatorname{dim} f\left(K^{m}\right)=m$. However, $f\left(K^{m}\right)$ is open in $Y^{\prime}$ and thus $\operatorname{dim} f\left(K^{m}\right)=n$, a contradiction. That is, $\operatorname{dim} B \leqq m-1$. Thus, $\operatorname{dim} f(B) \leqq m-1$. Now there is an $F$ closed in $Y^{\prime}$, hence in $Y$, with $\operatorname{dim} F \geqq n-m+1$ and $\operatorname{dim} F \cap f(B) \leqq 0$. Then $f \mid f^{-1}(F)$ satisfies the conditions of Theorem I.1. Thus there is a closed $K \subset f^{-1}(F)$ with $\operatorname{dim} K=0$ and $f(K)=F$. Clearly since $X$ is $\sigma$-compact we can require $K$ to be compact if we so desire.

II.11. REMARK. In this last proof we really only used the property that if $B C X$ with $\operatorname{dim} B=\operatorname{dim} X$, then $B$ has interior points in $X$. Thus, there is an obvious generalization of II.10 to spaces $X$ having this property. Many dimension theorems for manifolds could be generalized to such spaces.

ADDED IN PROOF. There are improvements of the main results of this paper in the author's dissertation (Mappings and dimension in metric spaces), University of Miami, June 1968. 


\section{REFERENCES}

1. P. Civin and J. H. Roberts, Sections of continuous collections, Bull. Amer. Math. Soc. 49 (1943), 142-143.

2. J. Dugundji, Topology, Allyn and Bacon, Boston, Mass., 1965.

3. R. Hodel, Open functions and dimension, Duke, Math. J. 30 (1963), 461-467.

4. J. Keesling, Mappings and dimension in general metric spaces, Pacific J. Math. 25 (1968), 277-288.

5. C. Kuratowski, Topologie. II, Monogr. Mat., Warsaw, 1950.

6. J. Nagata, Modern dimension theory, Wiley, New York, 1965.

UNIVERSITY OF MiAMI 